\title{
Path integral duality modified propagators in spacetimes with constant curvature
}

\author{
Dawood Kothawala ${ }^{1+}$, L. Sriramkumar ${ }^{2 \dagger}$, \\ S. Shankaranarayanan ${ }^{3 \ddagger}$ and T. Padmanabhan ${ }^{1 *}$ \\ ${ }^{1}$ IUCA A, Post Bag 4, Ganeshkhind, Pune 411 00\%, India. \\ ${ }^{2}$ Harish-Chandra Research Institute, Chhatnag Road, Jhunsi, Allahabad 211 019, India. \\ ${ }^{3}$ Institute of Cosmology and Gravitation, University of Portsmouth, \\ Mercantile House, Portsmouth P01 2EG, U.K.. \\ E-mail: ${ }^{+}$dawood@iucaa.ernet.in, ${ }^{\dagger}$ sriram@hri.res.in, \\ ${ }^{\ddagger}$ Shanki.Subramaniam@port.ac.uk, * paddy@iucaa.ernet.in
}

(March 10, 2019)

\begin{abstract}
The hypothesis of path integral duality provides a prescription to evaluate the propagator of a free, quantum scalar field in a given classical background, taking into account the existence of a fundamental length, say, the Planck length, $L_{\mathrm{P}}$, in a locally Lorentz invariant manner. We use this prescription to evaluate the duality modified propagators in spacetimes with constant curvature (exactly in the case of one spacetime, and in the Gaussian approximation for another two), and show that: (i) the modified propagators are ultra violet finite, (ii) the modifications are non-perturbative in $L_{\mathrm{P}}$, and (iii) $L_{\mathrm{P}}$ seems to behave like a 'zero point length' of spacetime intervals such that $\left\langle\sigma^{2}\left(x, x^{\prime}\right)\right\rangle=\left[\sigma^{2}\left(x, x^{\prime}\right)+\mathcal{O}(1) L_{\mathrm{P}}^{2}\right]$, where $\sigma\left(x, x^{\prime}\right)$ is the geodesic distance between the two spacetime points $x$ and $x^{\prime}$, and the angular brackets denote (a suitable) average over the quantum gravitational fluctuations. We briefly discuss the implications of our results.
\end{abstract}

\section{Motivation}

It is presumed that quantum gravitational effects would become important at length scales of the order of the Planck length, $L_{\mathrm{P}}=\left(G \hbar / c^{3}\right)^{1 / 2}$. At these scales, it seems quite likely that the description of the spacetime structure in terms of a metric, as well as certain notions of standard quantum field theory, would have to undergo drastic changes. Since any quantum field has virtual excitations of arbitrary high energy, which probe arbitrarily small scales, it follows that the conventional quantum field theory can only be an approximate description that is valid at energies smaller than the Planck energy.

The existence of a fundamental length implies that processes involving energies higher than the Planck energy can possibly be suppressed, thereby improving the ultra violet behavior of the theory. In particular, one hopes that, in a complete theory, gravity would provide an effective cut off at the Planck scales [1]. Some very general considerations based on the principle of equivalence and the uncertainty principle seem to strongly indicate that it may not be possible to operationally define spacetime events beyond an accuracy of the order of $L_{\mathrm{P}}$. Therefore, one may consider $L_{\mathrm{P}}$ as the 'zero point length' of spacetime intervals [2]. Specifically, if $\sigma\left(x, x^{\prime} \mid g_{\mu \nu}\right)$ denotes the geodesic distance between the spacetime points $x$ and $x^{\prime}$ in the background metric $g_{\mu \nu}$, then one can expect that

$$
\lim _{x \rightarrow x^{\prime}}\left\langle\sigma^{2}\left(x, x^{\prime} \mid g_{\mu \nu}+h_{\mu \nu}\right)\right\rangle=\mathcal{O}(1) L_{\mathrm{P}}^{2},
$$

where $h_{\mu \nu}$ represents all possible quantum fluctuations about the background metric, and the angular brackets represent a suitable path integral average over these fluctuations. Such a behavior can then be expected to render the coincidence limit of the propagators finite. 
Let us now briefly outline as to how such a result might arise in a simple example. Consider a toy model of quantum gravity, where one considers quantum fluctuations in the conformal factor of the metric of flat spacetime. In such a case

$$
g_{\mu \nu}(x)=[1+\varphi(x)]^{2} \eta_{\mu \nu}
$$

and one treats $\varphi(x)$ as the relevant degrees of freedom describing the spacetime, which must be quantized. (It is often claimed in literature that the conformal factor should not be regarded as a 'physical' degree of freedom. This is incorrect, and we have outlined the essential arguments in App. A.) It can be easily shown that [3]

$$
\left\langle\varphi(x) \varphi\left(x^{\prime}\right)\right\rangle=\frac{L_{\mathrm{P}}^{2}}{\sigma_{\mathrm{M}}^{2}\left(x, x^{\prime}\right)},
$$

where $\sigma_{\mathrm{M}}^{2}\left(x, x^{\prime}\right)$ is the Lorentz invariant interval in flat spacetime. We shall now explicitly illustrate how a result such as (1) might be obtained. We write down the Taylor series expansion of the Synge's world function, $\Omega\left(x, x^{\prime}\right)=\left[\sigma^{2}\left(x, x^{\prime}\right) / 2\right]$ around the base point, say, $x$, in terms of the parameter $s$. If we now take the vacuum expectation value $\lim _{x \rightarrow x^{\prime}}\left\langle\Omega\left(x, x^{\prime}\right)\right\rangle$, and use Eq. (3), each term of the expansion turns out to be proportional to $L_{\mathrm{P}}^{2}$. (Specifically, after point-splitting the resulting series, the $k^{\text {th }}$ term contains $(k-2)$ derivatives of the right hand side of Eq. (3), which is multiplied by $\left(x-x^{\prime}\right)^{k}$, thereby giving a finite coincidence limit.) In fact, an exact path integral average of the propagator, say, $G\left(x, x^{\prime}\right)$, over the quantum fluctuations of $\varphi(x)$ can be carried out for the special case of a conformally coupled scalar field [2]. The result obtained is

$$
\left\langle G\left[\sigma\left(x, x^{\prime}\right)\right]\right\rangle=G\left(\left\langle\sigma\left(x, x^{\prime}\right\rangle\right)\right.
$$

with $\left\langle\sigma\left(x, x^{\prime}\right)\right\rangle$ interpreted as in Eq. (5) below. These calculations suggest that Eqs. (1) and (5) might be valid in a more general context. As we shall see, the results we obtain in this paper indeed point to such a conclusion (as was originally suggested in Ref. [4]).

The points mentioned above are highly suggestive. However, to set them on a more firm basis, one must use a physically well motivated hypothesis that incorporates one or more of the effects expected to arise in the actual quantum theory of gravitation. It would then indeed be interesting to examine whether one could derive a connection between the results mentioned above and some general underlying principle. Perhaps, the best strategy would be to accept our ignorance of the Planck scale physics and use, at the semiclassical level, a prescription to obtain effective quantum field theoretic propagators which captures the resultant smearing of quantum fields over a region of size of the order of $L_{\mathrm{P}}$. One such prescription was outlined in an earlier work, wherein the invariance of the path integral for a free, relativistic particle, under a duality transformation which keeps $L_{\mathrm{P}}$ invariant (for more details, see the next section) was demanded, and the corresponding modifications to the two point function was worked out [4]. It was shown that, in the Minkowski spacetime, the hypothesis of path integral duality (PID) proves to be essentially equivalent to adding a zero point length to spacetime intervals, in the sense of Eq. (1) - a relation, which we should stress is not at all obvious. The PID approach has since been utilized to compute the possible quantum gravitational modifications to a variety of effects in flat as well as curved spacetimes $[5,6,7,8]$.

In this paper, we shall consider the PID modifications to the two point function of a quantum scalar field in spacetimes with constant scalar curvature. After a rapid outline of the PID prescription, we shall evaluate the modified Green's function in the Einstein static universe and the de Sitter as well as the anti-de Sitter spacetimes. We find that, in $(3+1)$-dimensions, while the modified propagator can be evaluated exactly in the Einstein static universe, it is not amenable to an exact calculation in the de Sitter and the anti-de Sitter spacetimes. Therefore, we compute the modified propagator in the Gaussian approximation in the latter two cases. In the case of the anti-de Sitter spacetime, it 
turns out that an exact evaluation of the modified propagator is possible in $(2+1)$-dimensions [8], an example which we shall also briefly consider. We shall show that, in all these cases, the modified propagator remains finite in the coincidence limit, and the modifications are non-perturbative in $L_{\mathrm{P}}$. The form of the modified propagators that we obtain suggests that, under the PID prescription, the geodesic distance $\sigma\left(x, x^{\prime} \mid g_{\mu \nu}\right)$ is modified as

$$
\left\langle\sigma^{2}\left(x, x^{\prime} \mid g_{\mu \nu}+h_{\mu \nu}\right)\right\rangle=\sigma^{2}\left(x, x^{\prime} \mid g_{\mu \nu}\right)+\mathcal{O}(1) L_{\mathrm{P}}^{2},
$$

in agreement with Eq. (1).

We shall work with the metric signature $(-,+,+, \ldots,+)$, and we shall set $\hbar$ and $c$ to unity. Also, we shall denote the set of coordinates $x^{\mu}$ as $x$. Moreover, for convenience, we shall hereafter refer to the geodesic distance $\sigma\left(x, x^{\prime} \mid g_{\mu \nu}\right)$ simply as $\sigma\left(x, x^{\prime}\right)$.

\section{The setup}

Let $G\left(x, x^{\prime}\right)$ denote the two point function associated with a quantized, free scalar field that is propagating in a classical gravitational background described by the metric tensor $g_{\mu \nu}$. The two point function can be expressed as a relativistic path integral with the action given by $\left[m \mathcal{R}\left(x, x^{\prime}\right)\right]$, where $m$ is the mass of the scalar field and $\mathcal{R}\left(x, x^{\prime}\right)$ is the proper length of the path linking the two spacetime points $x$ and $x^{\prime}$ (see, for instance, Ref. [3]). The hypothesis of PID suggests that the path integral amplitude in the sum over the paths be modified such that paths with lengths below the Planck scale are suppressed in the sum, while maintaining invariance under the duality transformation: $\mathcal{R} \rightarrow\left(L_{\mathrm{P}}^{2} / \mathcal{R}\right)$. The specific prescription being that, instead of the original sum over paths, viz.

$$
G\left(x, x^{\prime}\right)=\sum_{\text {all paths }} \exp i\left[m \mathcal{R}\left(x, x^{\prime}\right)\right],
$$

one considers the following [4]:

$$
G_{\text {PID }}\left(x, x^{\prime}\right)=\sum_{\text {all paths }} \exp i m\left[\mathcal{R}\left(x, x^{\prime}\right)+\frac{L_{\mathrm{P}}^{2}}{\mathcal{R}\left(x, x^{\prime}\right)}\right] .
$$

In the Minkowski spacetime, such a modified sum over the paths can be carried out exactly using lattice techniques [4]. For a massless scalar field, it can be shown that the modified propagator is given by

$$
G_{\mathrm{PID}}\left(x, x^{\prime}\right)=\left(\frac{i}{4 \pi^{2}}\right) \frac{1}{\sigma_{\mathrm{M}}^{2}\left(x, x^{\prime}\right)+L_{\mathrm{P}}^{2}},
$$

where, as we mentioned earlier, $\sigma_{\mathrm{M}}^{2}\left(x, x^{\prime}\right)$ denotes the Lorentz invariant, flat spacetime interval. Evidently, the above PID modified propagator is finite in the coincidence limit. Also, the form is indeed suggestive of the spacetime itself as having a minimal length scale of $\mathcal{O}\left(L_{\mathrm{P}}\right)$ - a feature, as we had discussed, that is expected to arise when we take the quantum gravitational effects into account. One must note that it is not a priori evident as to why there must exist any connection whatsoever between the PID invariance and the existence of a minimal length scale. Hence, as it has been stressed in earlier works, the result in Eq. (8) is non-trivial. But, we should add that a similar result arrived at using the T-duality in string theory seems to indicate towards the possibility of such a connection [9]. Returning to the modified propagator (8), we see that it is non-perturbative in $L_{\mathrm{P}}$. Clearly, one could not have obtained this result by a perturbation expansion in $L_{\mathrm{P}}$.

To apply the above results to curved spacetime, let us recall that the propagator $G\left(x, x^{\prime}\right)$ can also be expressed in Schwinger's proper time representation as follows [10, 11]:

$$
G\left(x, x^{\prime}\right)=i \int_{0}^{\infty} \mathrm{d} s K\left(x, x^{\prime} ; s\right)
$$


where $K\left(x, x^{\prime} ; s\right)$ is given by

$$
K\left(x, x^{\prime} ; s\right) \equiv\left\langle x|\exp -(i \hat{\mathcal{H}} s)| x^{\prime}\right\rangle
$$

The quantity $K\left(x, x^{\prime} ; s\right)$ is formally equivalent to the path integral amplitude for a quantum mechanical system described by the Hamiltonian

$$
\hat{\mathcal{H}}=-\left(\square-m^{2}-\xi R\right) \equiv-\frac{1}{\sqrt{-g}} \partial_{\mu}\left(\sqrt{-g} g^{\mu \nu} \partial_{\nu}\right)+m^{2}+\xi R
$$

with $\xi$ being the coefficient of non-minimal coupling and $R$ denoting the scalar curvature of the background spacetime. Note that, in the kernel $K\left(x, x^{\prime} ; s\right), s$ plays the role of the 'time' parameter (the so called 'proper time'). In flat spacetime, it was shown that PID modifies the expression (9) for the two point function to be [4]

$$
G_{\text {PID }}\left(x, x^{\prime}\right)=i \int_{0}^{\infty} \mathrm{d} s \mathrm{e}^{i L_{\mathrm{P}}^{2} / 4 s} K\left(x, x^{\prime} ; s\right) .
$$

As mentioned above, such a modification has also been derived in the context of string theory using T-duality [9]. While this connection is very suggestive, it is not a rigorous proof and, in this paper, we shall treat Eq. (12) as a prescription and explore the consequences.

In $(D+1)$ spacetime dimensions, the proper time kernel $K\left(x, x^{\prime} ; s\right)$ can be expressed in the Schwinger-DeWitt series representation as follows $[11,12,13,14]$ :

$$
K\left(x, x^{\prime} ; s\right)=i(4 \pi i s)^{-(D+1) / 2} \Delta^{1 / 2}\left(x, x^{\prime}\right) \mathrm{e}^{i \sigma^{2}\left(x, x^{\prime}\right) / 4 s} \sum_{n=0}^{\infty} a_{n}\left(x, x^{\prime}\right)(i s)^{n},
$$

where $\sigma^{2}\left(x, x^{\prime}\right)$ is the square of the proper distance along the geodesic between the spacetime points $x$ and $x^{\prime}$. The quantity $\Delta\left(x, x^{\prime}\right)$ is a biscalar that is defined as

$$
\Delta\left(x, x^{\prime}\right)=-[-g(x)]^{-1 / 2} \operatorname{Det}\left(-\frac{\partial^{2}\left[\sigma^{2}\left(x, x^{\prime}\right) / 2\right]}{\partial x^{\mu} \partial x^{\prime \nu}}\right)\left[-g\left(x^{\prime}\right)\right]^{-1 / 2}
$$

with $g(x)$ being the determinant of the metric tensor $g_{\mu \nu}$. It can be shown that the coefficients $a_{n}$ satisfy the recursion relation [14]

$$
a_{n}\left(x, x^{\prime}\right)=\sigma^{-n}\left(x, x^{\prime}\right) \int_{0}^{\sigma} \mathrm{d} \sigma(q) \sigma^{(n-1)}(q) \quad\left[\Delta^{-1 / 2} \square\left(\Delta^{1 / 2} a_{n-1}\right)\right]\left[x(q), x^{\prime}\right],
$$

where $\sigma(q)=\sigma\left[x(q), x^{\prime}\right]$, and the integral is along the geodesic connecting the spacetime points $x$ and $x^{\prime}$. In what follows, we shall use the above representation of the kernel in Eq. (12) to evaluate the PID modified propagator in spacetimes of constant curvature.

\section{The modified propagator in spacetimes with constant curvature}

Spacetimes with constant scalar curvature $R$ are maximally symmetric. Let $\ell$ be a constant that denotes the characteristic length scale associated with these spacetimes. Then, the metric describing these spacetimes can be expressed as (see, for instance, Ref. [15])

$$
\mathrm{d} s^{2}=g_{\mu \nu} \mathrm{d} x^{\mu} \mathrm{d} x^{\nu}=\eta_{\mu \nu} \mathrm{d} x^{\mu} \mathrm{d} x^{\nu}+\left(\frac{\mathcal{K} / \ell^{2}}{1-\left(\mathcal{K} / \ell^{2}\right) \eta_{\rho \sigma} x^{\rho} x^{\sigma}}\right)\left(\eta_{\mu \nu} x^{\mu} \mathrm{d} x^{\nu}\right)^{2},
$$

where the quantity $\mathcal{K}$ can take values $-1,0$ or 1 . The symmetries of these spacetimes-viz. homogeneity and isotropy about every point - are more evident in, for example, the spherical coordinates, and we shall employ these coordinates for our calculations. 


\subsection{The Einstein static universe}

In $(3+1)$ spacetime dimensions, the Einstein static universe $(\mathrm{ESU})$, corresponding to $\mathcal{K}=1$, can be described by the line element

$$
\mathrm{d} s^{2}=-\mathrm{d} t^{2}+\ell^{2}\left(\mathrm{~d} \chi^{2}+\sin ^{2} \chi \mathrm{d} \Omega_{2}^{2}\right)
$$

where $d \Omega_{2}^{2}$ denotes the metric on a 2 -dimensional unit sphere. ESU is topologically $\left(\mathbb{R} \times \mathbb{S}^{3}\right)$. The quantity $\ell$ in the above metric denotes the radius of $\mathbb{S}^{3}$, and it is related to the scalar curvature $R$ of the spacetime by the relation: $R=\left(6 / \ell^{2}\right)$. The kernel in the ESU can be factorized as

$$
K_{\left(\mathbb{R} \times \mathbb{S}^{3}\right)}\left(t, \mathbf{x}, t^{\prime}, \mathbf{x}^{\prime} ; s\right)=(-4 \pi i s)^{-1 / 2} \exp -i\left[\left(t-t^{\prime}\right)^{2} / 4 s\right] K_{\mathbb{S}^{3}}\left(\mathbf{x}, \mathbf{x}^{\prime} ; s\right),
$$

where $K_{\mathbb{S}^{3}}\left(\mathbf{x}, \mathbf{x}^{\prime} ; s\right)$ is the kernel corresponding to the coordinates $\mathbf{x}$ and $\mathbf{x}^{\prime}$ on $\mathbb{S}^{3}$. Now, for $\mathbb{S}^{3}$, it can be shown by direct computation that the relation (see, for example, Ref. [14])

$$
\Delta^{1 / 2}=(R / 6) \Delta^{1 / 2}
$$

holds. The coefficients $a_{n}$ 's can then be obtained from the recursion relation (15), and they turn out to be (with $\left.a_{0}=1\right): a_{n}=(1 / n !)(R / 6)^{n}$. As a result, the sum in Eq. (13) can be evaluated exactly to arrive at the following form for the kernel:

$$
K_{\mathbb{S}^{3}}\left(\mathbf{x}, \mathbf{x}^{\prime} ; s\right)=(4 \pi i s)^{-3 / 2} \Delta^{1 / 2}\left(\mathbf{x}, \mathbf{x}^{\prime}\right) \quad \exp i\left[\left(\sigma^{2}\left(\mathbf{x}, \mathbf{x}^{\prime}\right) / 4 s\right)+(R s / 6)\right],
$$

where $\sigma\left(\mathbf{x}, \mathbf{x}^{\prime}\right)$ is the geodesic distance on $\mathbb{S}^{3}$. Also, for $\mathbb{S}^{3}$, it can be easily shown that

$$
\Delta^{1 / 2}\left(\mathbf{x}, \mathbf{x}^{\prime}\right)=\left(\frac{\sigma\left(\mathbf{x}, \mathbf{x}^{\prime}\right) / \ell}{\sin \left[\sigma\left(\mathbf{x}, \mathbf{x}^{\prime}\right) / \ell\right]}\right)
$$

Therefore, upon using Eqs. (20) and (18) in (12), we obtain the PID modified Greens function in the ESU to be

$$
G_{\mathrm{PID}}\left(x, x^{\prime}\right)=-\left(\frac{\sqrt{b}}{8 \pi}\right) \sum_{n=-\infty}^{\infty}\left(\frac{H_{1}^{(2)}\left(\sqrt{b\left[u_{n}^{2}\left(x, x^{\prime}\right)-L_{\mathrm{P}}^{2}\right]}\right)}{\sqrt{u_{n}^{2}\left(x, x^{\prime}\right)-L_{\mathrm{P}}^{2}}}\right) \Delta_{n}^{1 / 2}\left(\mathbf{x}, \mathbf{x}^{\prime}\right),
$$

where $b=\left[m^{2}+(6 \xi-1)(R / 6)\right]$, and $H_{1}^{(2)}$ is the Hankel function of the second kind and order one. Also, $u_{n}^{2}\left(x, x^{\prime}\right)=\left[\left(t-t^{\prime}\right)^{2}-\sigma_{n}^{2}\left(\mathbf{x}, \mathbf{x}^{\prime}\right)\right]$, and the quantities with subscript $n$ are obtained by replacing $\sigma\left(\mathbf{x}, \mathbf{x}^{\prime}\right)$ with $\sigma_{n}\left(\mathbf{x}, \mathbf{x}^{\prime}\right)=\left[\sigma\left(\mathbf{x}, \mathbf{x}^{\prime}\right)+(2 \pi n) \ell\right]$. Since $\mathbb{S}^{3}$ is compact, the sum over $n$ in the above expression essentially takes into account geodesics with higher and higher windings that connect the two points $\mathbf{x}$ and $\mathbf{x}^{\prime}$, while the $n=0$ term corresponds to the contribution due to the direct path. In the standard case (i.e. when $L_{\mathrm{P}}=0$ ), it is the $n=0$ term that leads to divergences in the coincidence limit ${ }^{1}$.

\footnotetext{
${ }^{1}$ A quick way to see that the $n \neq 0$ terms are finite in the coincidence limit is to note that, after separating out the $n=0$ term and a series independent of $\sigma$, we are left with a term proportional to

$$
\csc (\sigma / \ell) \times \sum_{n \neq 0} n H_{1}^{(2)}\left[i \sqrt{b\left(\sigma_{n}^{2}+L_{\mathrm{P}}^{2}\right)}\right]\left(\sigma_{n}^{2}+L_{\mathrm{P}}^{2}\right)^{-1 / 2}
$$

in the modified Green's function. For $\sigma=0$, the sum multiplying $\csc (\sigma / \ell)$ is strictly zero since it is odd in $n$. Considered as a smooth function of $\sigma$, this sum must therefore have an expansion in positive powers of $\sigma$, with the leading term going as $\sigma$. Therefore, in the $\sigma \rightarrow 0$ limit, we are left with a finite contribution from this piece.
} 
Therefore, we concentrate on this term to demonstrate that the PID modified propagator is finite in the coincidence limit. Using the fact that $\Delta(\mathbf{x}, \mathbf{x})=1$, the $n=0$ term, in the coincidence limit $x \rightarrow x^{\prime}$, gives ${ }^{2}$

$$
G_{\mathrm{PID}}(x, x)=-\left(\frac{\sqrt{b}}{8 \pi i L_{\mathrm{P}}}\right) H_{1}^{(2)}\left(i \sqrt{b} L_{\mathrm{P}}\right) .
$$

We see that the divergent term has been rendered finite by $L_{\mathrm{P}}$. As $b \rightarrow 0$ (i.e. for the case of a massless and conformally coupled field), the modified propagator reduces to

$$
G_{\mathrm{PID}}(x, x)=\left(\frac{i}{4 \pi^{2}}\right) \frac{1}{L_{\mathrm{P}}^{2}} .
$$

It is clear from the form of the modification that $L_{\mathrm{P}}$ acts as a residual, zero point length of spacetime intervals. In the same limit, for $x \neq x^{\prime}$, the two point function can be expressed as

$$
G_{\mathrm{PID}}(x, x)=\left(\frac{i}{4 \pi^{2}}\right)\left(\frac{\Delta^{1 / 2}}{u_{0}^{2}}\right)\left(1+\frac{L_{\mathrm{P}}^{2}}{u_{0}^{2}}\right)^{-1} \approx\left(\frac{i}{4 \pi^{2}}\right) \Delta^{1 / 2}\left[\frac{1}{u_{0}^{2}}-\frac{L_{\mathrm{P}}^{2}}{u_{0}^{4}}+\cdots\right] .
$$

Each term in this expression diverges as $u \rightarrow 0$, and, as a result, the corrections are non-perturbative in $L_{\mathrm{P}}$. Moreover, under analytic continuation, $(\sigma / \ell) \rightarrow[i(\sigma / \ell)]$, and $\ell \rightarrow(i \ell)$, we can obtain the kernel and, hence, the propagator for $\left(\mathbb{R} \times \mathbb{H}^{3}\right.$ ) (there is, of course, no sum over $n$ now, since $\mathbb{H}^{3}$ is non-compact, and there is a unique geodesic connecting the two points). Clearly, all the above conclusions regarding the effects of $L_{\mathrm{P}}$ will apply to this case as well.

\section{2 de Sitter and anti de Sitter spacetimes}

The Euclidean continuation of the de Sitter spacetime is topologically $\mathbb{S}^{4}$, and the kernel can be obtained exactly in such a case (see, for instance, Ref. [16]). But, we find that the corresponding duality modified propagator can not be evaluated in a closed form using these expressions. So, we resort instead to the Gaussian approximation to the kernel [17]. Under this approximation, one constructs a Fermi normal coordinate system based on the extremal path, and considers only those paths in the path integral sum which deviate from the extremal path quadratically (in terms of appropriate Fourier coefficients). Therefore, as such, the approximation itself does not require the separations between the two spacetime points $x$ and $x^{\prime}$ to be small. However, as we shall show in App. B, for $\mathbb{S}^{N}$ the approximation becomes better for small geodesic separation between the points (which is the limit in which we are, eventually, interested).

In spacetimes of constant scalar curvature, under the Gaussian approximation, in $(3+1)$ dimensions, the kernel $K\left(x, x^{\prime} ; s\right)$ can be expressed as $[17]$

$$
K\left(x, x^{\prime} ; s\right) \simeq\left(\frac{i}{(4 \pi i s)^{2}}\right)(\operatorname{Det} D)^{-1 / 2} \exp i\left[\left(\sigma^{2}\left(x, x^{\prime}\right) / 4 s\right)-i(\xi R s / 6)\right] .
$$

The quantity Det $D$ is defined in [17], where it has been argued that, Det $D=\Delta^{-1}$ (this can be shown rigorously for ESU and de Sitter). For de Sitter, using the general result derived for arbitrary $\mathbb{S}^{\mathrm{N}}$ in the appendix, we see that,

$$
\square \Delta^{1 / 2} \simeq\left(\frac{R}{6}\right) \Delta^{1 / 2}-\left(\frac{1}{20}\right)\left(\frac{\sigma R}{12}\right)^{2} \Delta^{1 / 2}-\left[\mathcal{O}\left(\sigma^{4} \ell^{-6}\right)+\text { higher order terms }\right] .
$$

\footnotetext{
${ }^{2}$ The resemblance to the Minkowski space propagator for a massive field can be understood as follows. In the flat space limit, i.e. as $\ell \rightarrow \infty, \Delta^{1 / 2}=1$, as expected. Further, when $R$ is constant, the combination $\left(m^{2}+\xi R\right)$ can be looked upon as a effective 'mass' of the field.
} 
Hence, in the coincidence limit, $\square \Delta^{1 / 2} \sim(R / 6) \Delta^{1 / 2}$ is indeed a good approximation, which validates the use of the Gaussian approximation. To calculate the coincidence limit, we simply note that $\Delta(x, x)=1$. Thus, the coincidence limit of the propagator is the same as that for ESU, given by Eq. (23), and is therefore finite. Under analytic continuation, it is easy to see that the same result would hold for anti de Sitter $\left(\mathbb{H}^{4}\right)$ as well. In fact, for arbitrary $\mathbb{S}^{N}$, even though the exact form of the propagator would depend on $N$, we expect the coincidence limit to be finite. (If $\square \Delta^{1 / 2} \sim(R / 6) \Delta^{1 / 2}$ is not constant, it will act as a 'potential' and the extremal path will be accelerated. In this case, $(\text { Det } D)^{-1 / 2}$ becomes a complicated function involving $s$ [17], and (unfortunately!) the propagator can not be obtained in a closed form in general.)

As a final comment, let us mention that in Refs. [18], the modification $\sigma^{2} \rightarrow\left(\sigma^{2}+L_{\mathrm{P}}^{2}\right)$ was used in the context of cosmology (the motivation there being the results obtained earlier from quantization of the conformal factor of conformally flat metrics, and not path integral duality). It was found that with this modification, one can generate density perturbations of acceptable magnitude, and thereby make inflation work without any fine tuning of parameters. Our result for the de Sitter case may be of some use in such a context.

\subsection{Anti de Sitter spacetime in $(2+1)$-dimensions}

We shall now discuss the case of the $(2+1)$-dimensional anti de Sitter spacetime $\left(\mathrm{AdS}_{3}\right)$ separately, since it allows the exact evaluation of the kernel and the modified propagator.

Recently, we had computed the PID modifications to the propagator and the associated stressenergy tensor around the $(2+1)$-dimensional, Banados-Teitelboim-Zanelli (BTZ) black hole [8]. The BTZ black hole solution is obtained by discrete identifications of points on $\mathrm{AdS}_{3}$. Hence, we had required the kernel in $\mathrm{AdS}_{3}$ to arrive at the corresponding kernel around the BTZ black hole. We shall be brief here, since the details can be found in our earlier work.

$\mathrm{AdS}_{3}$ can be described by the line-element

$$
\mathrm{d} s^{2}=-\left(\frac{r^{2}}{\ell^{2}}-1\right) \mathrm{d} t^{2}+\left(\frac{r^{2}}{\ell^{2}}-1\right)^{-1} \mathrm{~d} r^{2}+r^{2} \mathrm{~d} \phi^{2},
$$

where $-\infty<(t, \phi)<\infty$ and $0<r<\infty$. The kernel in such a background can be easily evaluated using the method of spectral decomposition, and it is found to be

$$
K\left(x, x^{\prime} ; s\right)=\left(\frac{1}{(4 \pi i s)^{3 / 2}}\right)\left(\frac{\sigma\left(x, x^{\prime}\right) / \ell}{\sinh \left[\sigma\left(x, x^{\prime}\right) / \ell\right]}\right) \exp i\left[\left(\sigma^{2}\left(x, x^{\prime}\right) / 4 s\right)-\left(\beta s / \ell^{2}\right)\right],
$$

where $\beta=\left[1+(m \ell)^{2}+\xi R \ell^{2}\right], R=-\left(6 / \ell^{2}\right)$, and the quantity $\sigma\left(x, x^{\prime}\right)$ is given by

$\sinh \left[\sigma\left(x, x^{\prime}\right) / 2 \ell\right]=(\sqrt{2} \ell)^{-1}\left[-\sqrt{\left(r^{2}-\ell^{2}\right)\left(r^{\prime 2}-\ell^{2}\right)} \cosh \left[\left(t-t^{\prime}\right) / \ell\right]-\ell^{2}+r r^{\prime} \cosh \left(\phi-\phi^{\prime}\right)\right]^{1 / 2}$.

From the above kernel, the duality modified Green's function in $\mathrm{AdS}_{3}$ can be immediately obtained to be

$$
G_{\mathrm{PID}}\left(x, x^{\prime}\right)=\left(\frac{1}{4 \pi}\right)\left(\frac{\sigma\left(x, x^{\prime}\right) / \ell}{\sinh \left[\sigma\left(x, x^{\prime}\right) / \ell\right]}\right)\left(\frac{1}{\sqrt{\sigma^{2}\left(x, x^{\prime}\right)+L_{\mathrm{P}}^{2}}}\right) \exp -\left(\sqrt{\left(\beta / \ell^{2}\right)\left[\sigma^{2}\left(x, x^{\prime}\right)+L_{\mathrm{P}}^{2}\right]}\right) .
$$

In the coincidence limit, $\sigma\left(x, x^{\prime}\right) \rightarrow 0$, and this modified propagator reduces to

$$
G_{\mathrm{PID}}(x, x)=\left(\frac{1}{4 \pi L_{\mathrm{P}}}\right) \exp -\left(\sqrt{\beta} L_{\mathrm{P}} / \ell\right)
$$

which is, clearly, ultra violet finite. 


\section{Discussion}

We have analyzed the scalar field propagators in spacetimes of constant curvature, taking into account the Planck scale corrections according to the hypothesis of path integral duality. The main results can be summarized as follows:

1. In $(3+1)$-dimensions [cf. Eqs. $(22)$ and $(23)]$ :

$$
\begin{aligned}
G_{\mathrm{PID}}\left(x, x^{\prime}\right) & =-\left(\frac{\sqrt{b}}{8 \pi}\right)\left(\frac{H_{1}^{(2)}\left(\sqrt{b\left[u^{2}\left(x, x^{\prime}\right)-L_{\mathrm{P}}^{2}\right]}\right)}{\sqrt{u^{2}\left(x, x^{\prime}\right)-L_{\mathrm{P}}^{2}}}\right) \Delta^{1 / 2}\left(x, x^{\prime}\right) \\
\underset{x \rightarrow x^{\prime}}{\longrightarrow} & -\left(\frac{\sqrt{b}}{8 \pi i L_{\mathrm{P}}}\right) H_{1}^{(2)}\left(i \sqrt{b} L_{\mathrm{P}}\right),
\end{aligned}
$$

2. In $(2+1)$-dimensions [cf. Eqs. 31) and (32)]

$$
\begin{aligned}
& G_{\mathrm{PID}}\left(x, x^{\prime}\right)=\left(\frac{1}{4 \pi \ell}\right) \Delta^{1 / 2}\left(x, x^{\prime}\right)\left(\frac{\exp -\sqrt{b\left[u^{2}\left(x, x^{\prime}\right)-L_{\mathrm{P}}^{2}\right]}}{\sqrt{u^{2}\left(x, x^{\prime}\right)-L_{\mathrm{P}}^{2}}}\right) \\
& \underset{x \rightarrow x^{\prime}}{\longrightarrow}\left(\frac{1}{4 \pi L_{\mathrm{P}}}\right) \exp -\left(\sqrt{b} L_{\mathrm{P}}\right),
\end{aligned}
$$

where $u\left(x, x^{\prime}\right)$ represents the geodesic distance between the two points $x$ and $x^{\prime}$, and the other symbols have their meanings as stated in the text (multiple geodesics must be accounted for, as the case may be). We have therefore shown that, the hypothesis of PID, when applied to cases where the proper time kernels are known exactly (or under a suitable approximations): (i) regulates the theory at Planck scales, (ii) yields modifications which are non-perturbative in $L_{\mathrm{P}}$ and, (iii) most interestingly, we find that the quantum gravitational effects, as accounted for by the duality prescription, can be looked upon as leading to addition of $L_{\mathrm{P}}$ to all spacetime (geodesic) intervals in a (peculiar) Pythagorean way, that is, $\left\langle\sigma^{2}\left(x, x^{\prime}\right)\right\rangle=\left[\sigma^{2}\left(x, x^{\prime}\right)+\mathcal{O}(1) L_{\mathrm{P}}^{2}\right]$, as is clearly evident from the above expressions for the propagators.

The duality invariance of the relativistic point particle path integral is therefore equivalent to 'adding' a zero-point length to spacetime intervals, which is a non trivial point (as was already emphasized in [4]). Such a result might be an outcome of some generic short distance behavior of the spacetime structure itself, when quantum effects are taken into account. One expects that these results would naturally appear in the (effective) low energy sector of the full theory of quantum gravity.

\section{A Appendix: Conformal factor as a physical degree of freedom}

One often finds statements in literature that the conformal factor enters in the action with the 'wrong' sign in the kinetic term, and therefore must be unphysical. This is incorrect, as is easily seen from the following arguments.

Consider the general metric (for the sake of convenience, we shall work below in $(3+1)$-dimensions)

$$
\mathrm{d} s^{2}=-N^{2} \mathrm{~d} t^{2}+2 N_{j} \mathrm{~d} t \mathrm{~d} x^{j}+h_{i j} \mathrm{~d} x^{i} \mathrm{~d} x^{j},
$$

with the gauge choice, $N=\Omega, N_{j}=0$, and $h_{i j}=\left(\Omega^{2} \gamma_{i j}\right)$ (the metric components can, in general, depend on all the coordinates). In such a case, the metric reduces to

$$
\mathrm{d} s^{2}=\Omega^{2}\left(-\mathrm{d} t^{2}+\gamma_{i j} \mathrm{~d} x^{i} \mathrm{~d} x^{j}\right)=\Omega^{2} g_{\mu \nu} \mathrm{d} x^{\mu} \mathrm{d} x^{\nu} .
$$


Using the relation between the Ricci scalars of conformally related metrics, it is easy to show that the Einstein-Hilbert action can be written as

$$
S_{\mathrm{EH}}=\left(\frac{1}{16 \pi G}\right) \int \mathrm{d} t \mathrm{~d}^{3} x \sqrt{\gamma}\left[-6 \Omega \square_{g} \Omega+R\right],
$$

where $\square_{g} \Omega=\left(-\partial_{t}^{2} \Omega+\gamma^{i j} \partial_{i} \partial_{j} \Omega\right)$, and $R$ is the Ricci scalar of the metric $g_{\mu \nu}$. The action can be rewritten as

$$
\begin{aligned}
S_{\mathrm{EH}} & =\left(\frac{1}{16 \pi G}\right) \int \mathrm{d} t \mathrm{~d}^{3} x \sqrt{\gamma}\left[6 \Omega \partial_{t}^{2} \Omega-6 \Omega \gamma^{i j} \partial_{i} \partial_{j} \Omega+R\right] \\
& =\left(\frac{1}{16 \pi G}\right) \int \mathrm{d} t \mathrm{~d}^{3} x \sqrt{\gamma}\left[-6\left(\partial_{t} \Omega\right)^{2}+\ldots\right]
\end{aligned}
$$

where we have exhibited only the kinetic term associated with $\Omega$. We immediately see that the kinetic term has the wrong sign, which is the reason why the conformal degree of freedom is often regarded as unphysical.

That this is incorrect can be easily seen by choosing instead the gauge, $N=1, N_{j}=0$ and $h_{i j}=\left[F^{2}(x) \gamma_{i j}\right]$ in Eq. (33), so that the metric becomes

$$
\mathrm{d} s^{2}=-\mathrm{d} t^{2}+F^{2} \gamma_{i j} \mathrm{~d} x^{i} \mathrm{~d} x^{j}
$$

In this case, $F$ generates a class of metrics with 3-geometries conformal to $\gamma_{i j}$, which is chosen to have, say, Det $\gamma=$ constant. Now, we know that, the components of the 3 -metric definitely correspond to physical degrees of freedom. However, explicitly calculating the action, we find that

$$
S_{\mathrm{EH}}=\left(\frac{1}{16 \pi G}\right) \int \mathrm{d} t \mathrm{~d}^{3} x \sqrt{\gamma}\left[-6 F\left(\partial_{t} F\right)^{2}+\ldots\right] .
$$

The kinetic term corresponding to $F$ again appears with the wrong sign, even though we know that $F$ in this case corresponds to a physical degree of freedom. This clearly illustrates that the conformal factor is not an unphysical degree of freedom.

\section{B Appendix: $\square \Delta^{1 / 2}$ for $\mathbb{S}^{\mathrm{N}}$}

For a unit $N$-sphere, the Van-Vleck determinant is given by [14]

$$
f(\sigma) \equiv \Delta\left(x, x^{\prime}\right)^{1 / 2}=\left(\frac{\sin \sigma\left(x, x^{\prime}\right)}{\sigma\left(x, x^{\prime}\right)}\right)^{(N-1) / 2}
$$

(For $\mathbb{H}^{\mathrm{N}}, \Delta^{1 / 2}=(\sinh \sigma / \sigma)^{(N-1) / 2}$; all the steps below go through, with a few sign changes, while the final expression remains the same.) We begin by writing the $\square$ operator in Riemann normal coordinates as (see, for example, Ref. [12])

$$
\square=\frac{\partial^{2}}{\partial \sigma^{2}}+\left[\frac{\partial}{\partial \sigma}\left(\ln \Delta^{-1}\right)+\frac{N-1}{\sigma}\right] \frac{\partial}{\partial \sigma}
$$

It then follows that

$$
\square f(\sigma)=\frac{(N-1)(N-3)}{4}\left[\frac{1}{\sigma^{2}}-\frac{1}{\sin ^{2} \sigma}+\frac{N-1}{N-3}\right] f(\sigma)
$$


We now expand the terms within the square bracket in the limit $\sigma \rightarrow 0$, and obtain

$$
\begin{aligned}
\square f(\sigma) & =\frac{(N-1)(N-3)}{4}\left[\frac{2 N}{3(N-3)}-\frac{\sigma^{2}}{15}-\mathcal{O}\left(\sigma^{4}\right)\right] f(\sigma) \\
& =\left[\frac{N(N-1)}{6}-\frac{(N-1)(N-3)}{60} \sigma^{2}-\mathcal{O}\left(\sigma^{4}\right)\right] f(\sigma) \\
& =\left(\frac{R}{6}\right) f(\sigma)+\mathcal{O}\left(\sigma^{2 k}\right) \quad(k=1,2, \ldots) .
\end{aligned}
$$

In the last step, we have used the fact that, for $\mathbb{S}^{N}$ of unit radius, $R=[N(N-1)]$. Note that all terms of $\mathcal{O}\left(\sigma^{2}\right)$ and higher vanish for $N=3$, which is the case encountered in $\operatorname{ESU}\left(\mathbb{R} \times \mathbb{S}^{3}\right)$. The Gaussian approximation is therefore exact for $\mathbb{S}^{3}$ [17]. This result suggests generalizations of arguments used for ESU and de Sitter in $(3+1)$-dimensions, to arbitrary $\mathbb{S}^{\mathrm{N}}$ or $\mathbb{H}^{\mathrm{N}}$.

\section{References}

[1] B. S. DeWitt, Phys. Rev. Lett. 13, 114 (1964).

[2] T. Padmanabhan, Ann. Phys. (N.Y.) 165, 38 (1985); Class. Quantum Grav. 4, L107 (1987).

[3] J. V. Narlikar and T. Padmanabhan, Gravity, Gauge Theories, and Quantum Cosmology, (Springer, Berlin, 1986).

[4] T. Padmanabhan, Phys. Rev. Lett. 78, 1854 (1997); Phys. Rev. D 57, 6206 (1998).

[5] K. Srinivasan, L. Sriramkumar and T. Padmanabhan, Phys. Rev. D 58, 044009 (1998).

[6] S. Shankaranarayanan and T. Padmanabhan, Int. J. Mod. Phys. D 10, 351 (2001).

[7] L. Sriramkumar and S. Shankaranarayanan, JHEP 0612, 050 (2006).

[8] D. A. Kothawala, S. Shankaranarayanan and L. Sriramkumar, JHEP 0809, 095 (2008).

[9] M. Fontanini, E. Spallucci and T. Padmanabhan, Phys. Lett. B 633, 627 (2006); A. Smailagic, E. Spallucci and T. Padmanabhan, arXiv:hep-th/0308122.

[10] J. Schwinger, Phys. Rev. 82 (1951), 664.

[11] B. S. DeWitt, Phys. Rep. 19 (1975), 297.

[12] L. Parker, in Recent Developments in Gravitation, Eds. M. Levy and S. Deser (Plenum, New York, 1979).

[13] N. D. Birrell and P. C. W. Davies, Quantum Fields in Curved Space (Cambridge University Press, Cambridge, England, 1982), Sec. 3.6.

[14] R. Camporesi, Phys. Reps. 196, 1 (1990).

[15] S. Weinberg, Gravitation and Cosmology: Principles and Applications of the General Theory of Relativity (John Wiley and Sons, New York, 1972), Chap. 13.

[16] J. S. Dowker and R. Critchley, Phys. Rev. D 13, 3224 (1976).

[17] J. D. Bekenstein and L. Parker, Phys. Rev. D 23, 2850 (1981).

[18] T. Padmanabhan, Phys. Rev. Lett. 60, 2229 (1988); T. Padmanabhan, T. R. Seshadri, T. P. Singh, Phys. Rev. D 39, 2100 (1989). 